\title{
LA AUDITORÍA EN LA ADMINISTRACIÓN DE FONDOS PÚBLICOS
}

\author{
AUDIT IN THE MANAGEMENT OF PUBLIC FUNDS
}

\section{Julio V. Flores KonJA*}

Docente Principal de la Facultad de Ciencias Contables, UNMSM

Juan G. Miñano Lecaros ${ }^{* *}$

Docente Asociado de la Facultad de Ciencias Contables, UNMSM

[Recepción: Junio de 2010 / Conformidad: Julio de 2010]

\section{RESUMEN}

De acuerdo al artículo 770 de la Constitución Política del Perú, "La administración económica y financiera del Estado se rige por el presupuesto que anualmente aprueba el congreso. La estructura del presupuesto del sector público contiene dos secciones: gobierno central e instancias descentralizadas. El presupuesto asigna equitativamente los recursos públicos. Su programación y ejecución responden a los criterios de eficiencia, de necesidades sociales básicas y de descentralización”3.

Como se podrá apreciar, el Estado administra un flujo de efectivo, denominado "Fondos Públicos", el mismo que es el soporte cuantitativo del presupuesto institucional. Tratar de un presupuesto de ingresos y otro de gastos, es inexorablemente relacionar también de la existencia de un presupuesto cualitativo. Es decir, existen objetivos y metas que cumplir por las entidades públicas, pero estas no se podrían cumplir sin la existencia de un presupuesto cuantitativo. Este artículo tiene por objetivo plantear ciertas dificultades así como plantear soluciones del tema del Presupuesto cuantitativo.

Palabras clave: Gestión pública, fondos públicos, auditorías de fondos públicos, administración de fondos públicos.

\begin{abstract}
According to Article 77 of the Constitution of Peru, "The economic and financial management of the State is governed by the budget approved annually by Congress. The structure of public sector budget contains two sections: the central government and decentralized agencies. The budget allocates public resources equitably. The programming and implementation meet the criteria of efficiency, basic social needs and decentralization".

As you can see, the State manages cash flow, called "Public Funds", the same which is the quantitative support the institutional budget. Try a budget of income and other expenses, is also inextricably linked to the existence of a qualitative budget. That is, there are objectives and goals to achieve public entities, but these could not be met without the existence of a quantitative budget. This article has as objetive to roise certain difficulties and to propose solutions to the issue of quantitative budget.
\end{abstract}

Key words: Public management, public funds, public finance audits, management of public funds.

* Doctor en Ciencias Contables, UNMSM; Contador Público Colegiado Certificado. Docente Investigador de la Facultad de Ciencias Contables, UNMSM. E-mail:juliofloreskonja@hotmail.com

** Magíster en Ciencias Contables, UNMSM. Contador Público Colegiado Certificado. Docente Investigador de la Facultad de Ciencias Contables, UNMSM. E-mail: minano1969@hotmail.com

1 Constitución Política del Perú, artículo 77², Imprenta de los Talleres Gráficos de la Editora “Acuario", Lima, Perú, Edición 2008, página 14. 


\section{INTRODUCCIÓN}

Desde el punto de vista contable, existe primero un origen de fondos y luego la aplicación del mismo, contabilizándose como ingresos y gastos. Sin embargo, la administración de dichos fondos públicos, exige la previa comprensión de la estructura de la administración de fondos públicos.

En primer orden, debemos precisar de la existencia de una trilogía básica del control de fondos públicos, la misma que posee una estructura sostenida por tres elementos básicos, reflejados en normas, las cuales tienen que ser permanentemente revisadas para presentar su actualización correspondiente.

El primer componente lo conforma la Gestión Presupuestaria, que a mérito de la Ley N. ${ }^{\circ}$ 28411, "Ley General del Sistema Nacional del Presupuesto"2, se cuenta con el documento técnico - doctrinario, que permite, entre otros, administrar correctamente los fondos públicos en base a normas presupuestarias. A este primer componente, se agrega la "Ley de Presupuesto del Sector Público para el año fiscal 2010" (Ley N. $\left.{ }^{\circ} 29465\right)^{3}$, la Directiva N. ${ }^{\circ}$ 003-07-EF/76.01, Directiva para la Ejecución Presupuestaria y Anexos por Nivel de Gobierno Nacional, Gobierno Regional y Gobierno Local, aprobada vía Resolución Directoral N. ${ }^{\circ}$ 003-07-EF/76.014, así como también la Directiva de Tesorería, aprobada mediante Resolución Directoral N. ${ }^{\circ} 002-07-E F / 77.15^{5}$.

\section{GESTIÓN CONTABLE}

Este conjunto de normas son las guías que permiten una adecuada administración de los fondos públicos, es decir, son las guías normativas para la correcta administración del flujo de efectivo en la entidad, conociéndose, en primer lugar, el origen del mismo y, posteriormente, realizándose su aplicación, siendo el equivalente en conocer los ingresos de fondos y luego realizar el gasto del mismo.

El segundo componente, lo conforma la Gestión Contable, que obviamente deviene de la administración del flujo de efectivo (ingresos y gastos) que anteriormente hemos tratado, agregando aplicaciones contables como: provisión de cobranza dudosa, liquidación de rendición de cuenta de anticipos concedidos, etc.

Para la gestión contable, se recurre a los llamados instructivos contables, emanados de la Dirección Nacional de Contabilidad Pública, que a mérito del artículo $42^{\circ}$ de la Ley N. ${ }^{\circ} 28112$ "Ley marco de la administración financiera del sector público" ${ }^{6}$, es el ente rector del Sistema Nacional de Contabilidad. A la fecha se han aprobado veintitrés instructivos contables, el resultado presupuestario y financiero de la gestión pública anual, es reflejado mediante la Cuenta General de la República y que la Ley N.2 28708, "Ley General del Sistema Nacional de Contabilidad", en sus artículos $23^{\circ}$ al $35^{\circ}$, da las pautas necesarias para el cumplimiento de la obligación

2 Ley N. ${ }^{\circ} 28411$, Ley General del Sistema Nacional de Presupuesto, Boletín de Normas Legales del Diario Oficial El Peruano, Lima. 8 de diciembre de 2004, pp. 281887 al 281901.

3 Ley N.o 29465, Ley de Presupuesto del Sector Público para el año fiscal 2010, Boletín de Normas Legales del Diario Oficial El Peruano, Lima, 10 de diciembre de 2009, pp. 407433 al 407450.

4 Resolución Directoral N.` 003-2007-EF/76.01, aprueban Directiva N.` 003-2007-EF/76.01 "Directiva para la Ejecución Presupuestaria y Anexos por Nivel de Gobierno Nacional, Gobierno Regional y Gobierno Local”, Boletín de Normas Legales del Diario Oficial El Peruano, Lima, 21 de enero de 2007, pp. 338019 al 338070.

5 Resolución Directoral N. 002-2007-EF/77.15, aprueban Directiva de Tesorería, Boletín de Normas Legales del Diario Oficial El Peruano, Lima, 27 de enero de 2007, pp. 338443 al 338462.

6 Ley N.o 28112, Ley Marco de la Administración Financiera del Sector Público, Boletín de Normas Legales del Diario Oficial El Peruano, Lima, 28 de noviembre de 2003, pp. 256077. 
de informar por parte de los gestores de las administraciones públicas ${ }^{7}$.

La gestión presupuestaria que deviene en la administración de un flujo de efectivo, por la naturaleza misma del ingreso y gasto, ello, obviamente será el reflejo de la gestión pública, es expuesto en los Estados Financieros. En tal sentido, se realizará la auditoría y demás acciones de control sobre la gestión institucional.

De acuerdo al artículo $82^{\circ}$ de la Constitución Política del Perú, "la Contraloría General de la República, es una entidad descentralizada de Derecho Público que goza de autonomía conforme a su ley orgánica. Es el órgano superior del Sistema Nacional de Control, supervisa la legalidad de la ejecución del presupuesto del estado, de las operaciones de la deuda pública y de los actos de las instituciones sujetas a control"8; en tal sentido, como tercer componente, se encuentra el control gubernamental.

De acuerdo a la Ley N. ${ }^{\circ} 27785$, "Ley Orgánica del Sistema Nacional de Control y de la Contraloría General de la República”, la Contraloría General de la República, como ente rector según el artículo $13^{\circ}$ de la citada norma, realiza el control gubernamental, siendo el sistema que se encuentra conformado por los auditores de la propia Contraloría, los auditores internos de los pliegos presupuestarios y las sociedades de auditoría independientes, previamente designadas por la Contraloría. Uno de los objetivos del control gubernamental es evaluar el grado de rendimiento y transparencia en el uso de los fondos públicos, así como también evaluar el grado de legalidad de la información presupuestaria y financiera?

\section{LA ESTRUCTURA DE LA GESTIÓN PÚBLICA}

Según el artículo 20, titulado "ámbito de aplicación" de la Ley N. ${ }^{\circ} 28411$, "Ley General del Sistema Nacional de Prosupuesto", es aplicable dicha normativa a las entidades públicas, clasificadas en:

a) Entidades del Gobierno General a.1. Gobierno Nacional

a.1.1. Administración Control. Organismos representantes de los Poderes Ejecutivo, Legislativo y Judicial, y sus organismos públicos descentralizados, universidades públicas y organismos constitucionalmente autónomos

a.1.2. Organismos Reguladores

a.1.3. Organismos Recaudadores y Supervisores

a.1.4. Fondos Especiales con Personería Jurídica

a.1.5. Beneficencias

a.2. Gobierno Regional

a.2.1. Los Gobiernos Regionales y sus organismos públicos descentralizados

a.3. Gobierno Local

a.3.1. Los Gobiernos Locales y sus organismos públicos descentralizados

7 Ley N. 28708 , Ley General del Sistema Nacional de Contabilidad, Boletín de Normas Legales del Diario Oficial El Peruano, Lima, 12 de abril de 2006, pp. 316581 al 316586.

8 Constitución Política del Perú, artículo 82², Imprenta de los Talleres Gráficos de la Editora “Acuario", Lima, Perú, Edición 2008, p. 15.

9 Ley N.o 27785, "Ley Orgánica del Sistema Nacional de Control y de la Contraloría General de la República”, Lima, Boletín de Normas Legales del Diario Oficial El Peruano, 23 de julio de 2002, pp. 226885 al 226893. 


\section{La trilogía básica del control de fondos públicos}

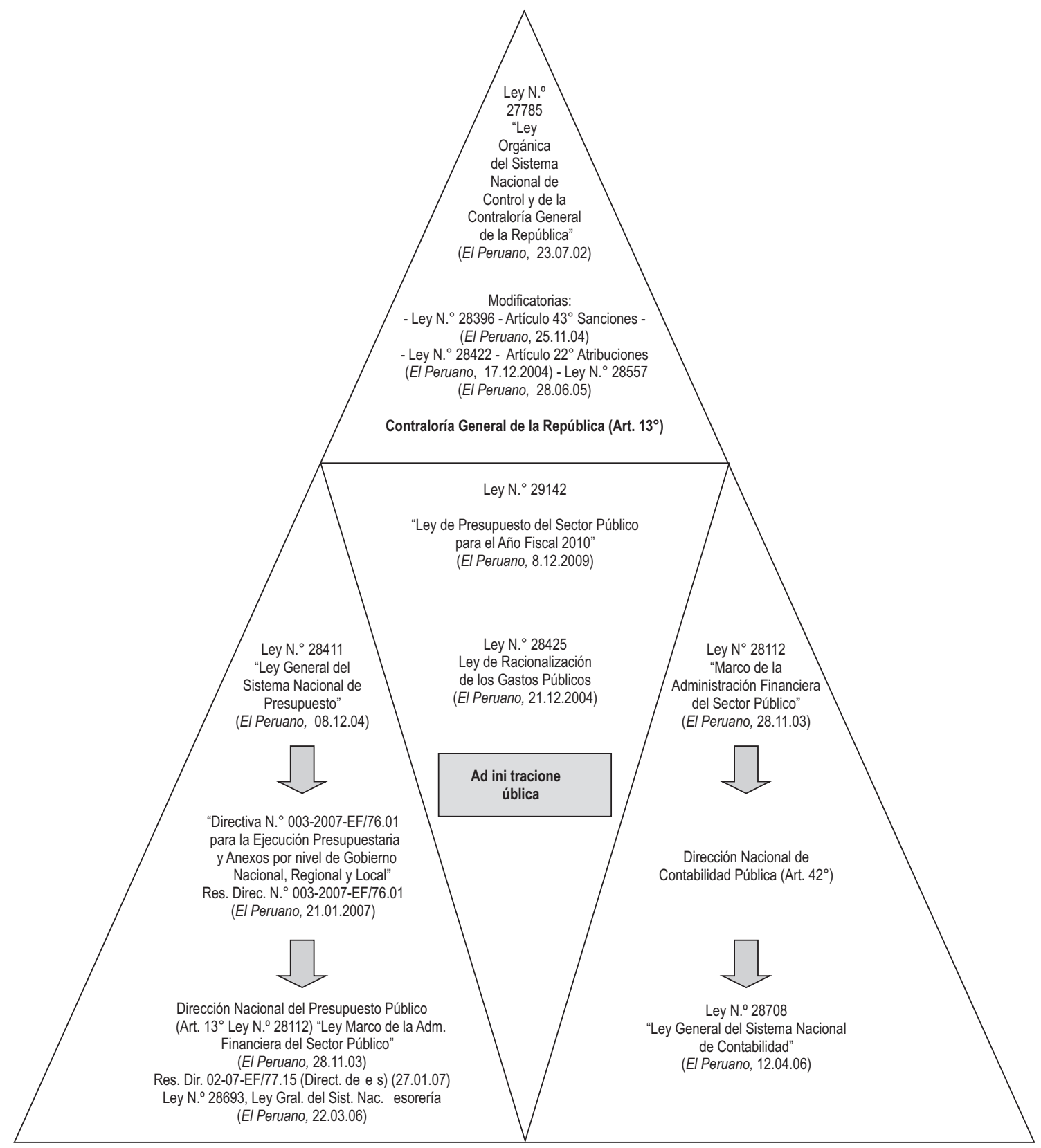

"Ley General del Sistema Nacional de Contabilidad" (El Peruano, 12.04.06) 
b) Empresas de los Gobiernos Regionales y Gobiernos Locales.

c) Fondos sin personería jurídica financiado total o parcialmente con fondos públicos.

d) Organismos carentes de personería jurídica con asignación presupuestaria.

e) El Fondo Nacional de Financiamiento de la Actividad Empresarial del Estado y sus empresas ${ }^{10}$.

Se puede apreciar una estructura de orden presupuestario, para la adecuada gestión y control gubernamental. Sin embargo, veamos la forma de presentación según la Ley N. 27785 , "Ley Orgánica del Sistema Nacional de Control y de la Contraloría General de la República", en lo que refiere al ámbito de aplicación de dicha norma, el artículo $3^{\circ}$ prescribe:

a) El Gobierno Central, sus entidades y órganos que forman el Poder Ejecutivo, incluyendo las FF.A.A. y Policía Nacional.

b) Los Gobiernos Regionales y Locales, instituciones y empresas.

c) Las unidades administrativas del Poder Legislativo, Judicial y del Ministerio Público.

d) Organismos Autónomos Constitucionales, instituciones y personas de derecho público.

e) Organismos reguladores de los servicios públicos y entidades supervisoras de los contratos de privatización.

f) Empresas del estado.

g) Entidades privadas, no gubernamentales e internacionales, por los recursos del Estado administrado ${ }^{11}$.
Hemos apreciado una estructura presupuestaria para los fines del control gubernamental, que obviamente se refiere a la definición de que el Estado administra un presupuesto.

La óptica estructural desde la perspectiva de Tesorería, la podemos apreciar en el artículo $2^{\circ}$ de la Ley N. ${ }^{\circ} 28693$, Ley General del Sistema Nacional de Tesorería, que prescribe de la siguiente manera:

a) Todas las entidades y organismos integrantes del Gobierno Nacional, Regional y Local.

b) Empresas del Estado de Derecho Público, Privado y Economía Mixta ${ }^{12}$.

Una estructura del Estado bastante comprimida, obviamente relacionada a la aplicación de la normativa sobre Tesorería, que es sencillamente el flujo del efectivo que se administra presupuestalmente.

Por otro lado, la Ley N. 28112 "Ley marco de la administración financiera del Sector Público", en su artículo $2^{\circ}$ "alcance”, presenta la siguiente estructura:

a) Organismos y entidades del Poder Legislativo, Ejecutivo y Judicial; así como el Ministerio Público.

b) Organismos conformantes del Sistema Nacional de Elecciones, el Consejo Nacional de la Magistratura, la Defensoría del Pueblo, Tribunal Constitucional, Contraloría General de la República, Universidades Públicas y entidades descentralizadas.

c) Gobiernos Regionales.

d) Gobiernos Locales y sus entidades descentralizadas.

10 Ley N.o 28411, "Ley General del Sistema Nacional de Presupuesto", Boletín de Normas Legales del Diario Oficial El Peruano, Lima, 8 de diciembre de 2004, pp. 281887.

11 Ley N.o 27785, "Ley Orgánica del Sistema Nacional de Control y de la Contraloría General de la República", Boletín de Normas Legales del Diario Oficial El Peruano, Lima, 23 de julio de 2002, pp. 226885.

12 Ley N.o 28693, "Ley General del Sistema Nacional de Tesorería", Boletín de Normas Legales del Diario Oficial El Peruano, Lima, 22 de marzo de 2006, pp. 315174. 


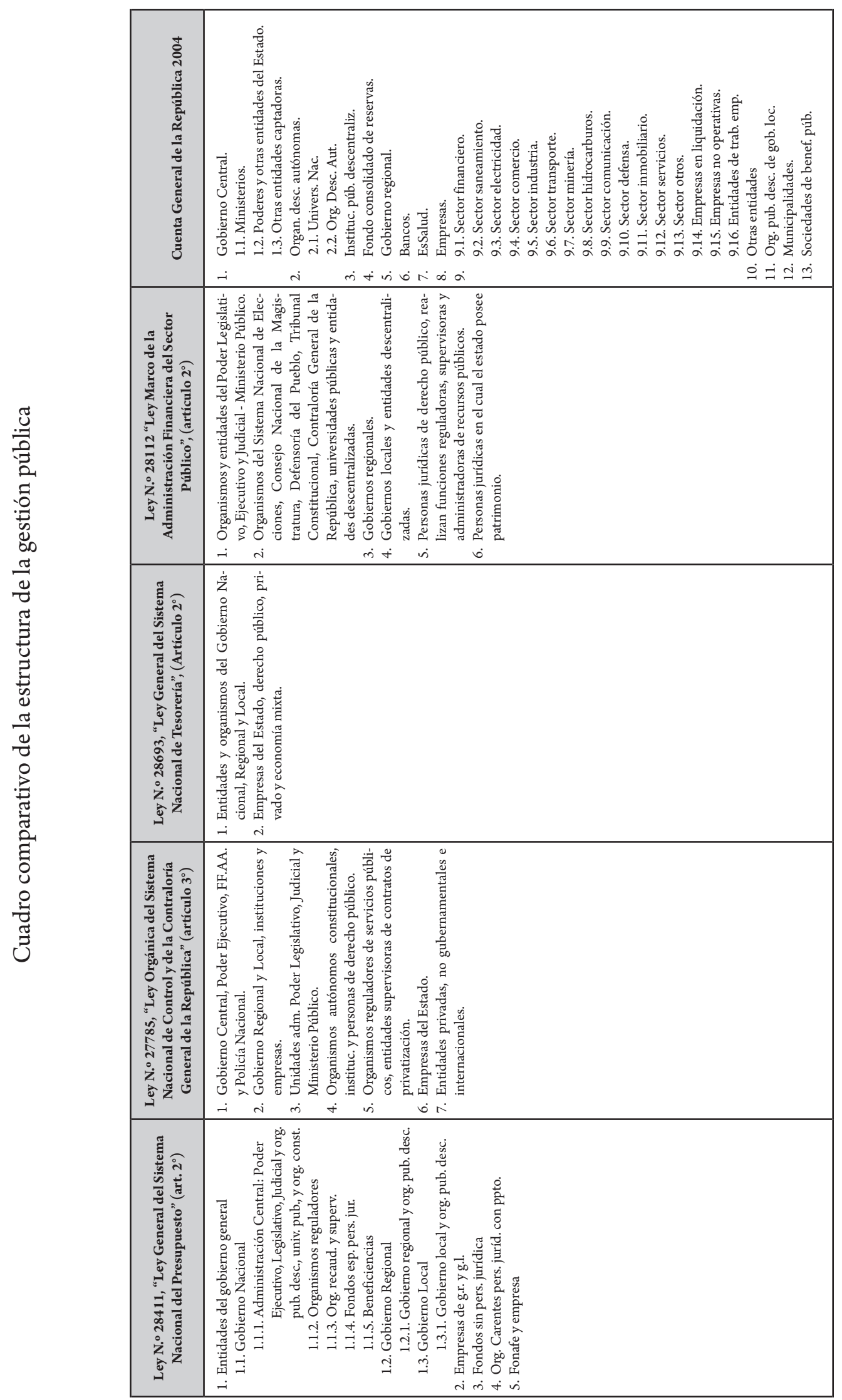


e) Personas Jurídicas de Derecho Público, con patrimonio propio que ejercen funciones reguladoras, supervisoras y administradores de recursos públicos.

f) Toda persona jurídica, donde el Estado posea Patrimonio ${ }^{13}$.

También se aprecia una estructura de carácter presupuestario, lo que colige con el concepto constitucional consagrado en el artículo $77^{\circ}$ de la Constitución Política del Perú, que define "El Estado administra un presupuesto".

A nivel de Cuenta General de la República, se aprecia una estructura de tipo tradicional, pues la información financiera y presupuestaria está organizada según: Gobierno Central, Organismos Descentralizados Autónomos, Instituciones Públicas Descentralizadas, Fondo Consolidado de Reservas, Gobierno Regional, Bancos, EsSalud, Empresas, Otras Entidades, Organismos Públicos Descentralizados del Gobierno Local, Municipalidades y Sociedades de Beneficencia Pública, tal como prescribe la Ley N. ${ }^{\circ}$ 28708, "Ley General del Sistema Nacional de Contabilidad”.

\section{LOS SISTEMAS ADMINISTRATIVOS}

Según la Ley N. ${ }^{\circ} 28112$, Ley marco de la administración financiera del Sector Público, existen sistemas con facultades y competencias de Ley y demás normas específicas para establecer procedimientos y directivas necesarias para su funcionamiento y operatividad $^{14}$.

La integración intersistémica de los sistemas de presupuesto, tesorería y contabilidad, permite que el desarrollo de los actos de gestión en las entidades públicas, sean reflejadas en la documentación pertinente, constituyéndose además, en la evidencia material y sustantiva.

En tal sentido, la administración del presupuesto institucional, que es también la administración de un flujo de efectivo, está condicionada básicamente a operaciones de ingresos y gastos, lo que tendrá su incidencia en la aplicación de normas de Tesorería, de acuerdo a la naturaleza de los actos de gestión y posteriormente es contabilizado, para su exposición en los Estados Financieros.

\section{De presupuesto}

De acuerdo al artículo $11^{\circ}$ y $12^{\circ}$ de la Ley N. 28112 , Ley marco de la administración financiera del sector público, el sistema nacional de presupuesto es el conjunto de órganos, normas y procedimiento que conducen el proceso presupuestario de todas las entidades y organismos del sector público en sus fases de programación, formulación, aprobación, ejecución y evaluación. Se rige por los principios de equilibrio, universalidad, unidad, especificidad, exclusividad y anualidad. El sistema se encuentra integrado por la Dirección Nacional del Presupuesto Público y las Oficinas de Planeamiento y Presupuesto de las entidades públicas ${ }^{15}$.

La característica fundamental del presupuesto público es su vigencia anual, el mismo que se inicia el primero de enero y concluye el 31 de diciembre, es decir, posee una característica anual.

13 Ley N.o 28112, "Ley Marco de la Administración Financiera del Sector Público", Boletín de Normas Legales del Diario Oficial El Peruano, Lima, 28 de noviembre de 2003, pp. 256074.

14 Ibídem, al anterior, artículo $4^{\circ}$, p. 256074.

15 Ibídem al anterior, artículo $11^{\circ}$ y $12^{\circ}$, p. 256075. 
Por otro lado, el presupuesto en el sector público, identifica plenamente el ingreso del egreso. Situación, que para lo primero, deben darse las etapas de estimación, determinación y percepción. Siendo el primero un cálculo o proyección, lo segundo, la identificación y oportunidad del ingreso, y lo tercero, la materialización del ingreso en la entidad.

Con respecto al gasto, sus etapas son: el compromiso, que es una afectación preventiva del presupuesto de la entidad, el devengado, es la recepción conforme del bien o servicio a ser adquirido y el pago, es la cancelación del compromiso ineludible de gasto.

Mediante Ley N. ${ }^{\circ} 28411$, Ley General del Sistema Nacional de Presupuesto, se posee los principios regulatorios y las disposiciones generales para la gestión presupuestaria que deben de aplicar el universo de entidades públicas ${ }^{16}$.

Para la aplicación de los controles internos a la gestión presupuestaria institucional, el ente rector del Sistema Nacional de Control, mediante Resolución de Contraloría $\mathrm{N}$. 072-98-CG, aprobó las "Normas Técnicas de Control Interno para el Sector Público", y fueron aplicables las normas: 200, "Normas de Control Interno para la administración financiera gubernamental"; norma 210, "Normas de control interno para el área de presupuesto", cuyo contenido es el siguiente:

a) Norma 210-01: Control del Marco Presupuestario de cada entidad.

b) Norma 210-02: Controles para la Programación de la Ejecución Presupuestaria.

c) Norma 210-03: Control previo de los compromisos presupuestales.

d) Norma 210-04: Control de la Evaluación del Presupuesto ${ }^{17}$.
Mediante Resolución de Contraloría N. ${ }^{\circ}$ 320-2006-CG, se derogan las Normas de Control Interno anteriormente indicadas, estando vigente en la actualidad las Normas Generales para la actividad de control gerencial, debiendo la entidad pública elaborar su propia regulación de control interno relacionada al ámbito presupuestario. Estas normas están expuestas en cinco grandes rubros: $\mathrm{El}$ primero, relacionado al ambiente de control, siendo su estructura y los recursos humanos su pilar. El segundo, se relaciona a la evaluación de los riesgos, en donde su identificación y valoración permiten una evaluación confiable. Como tercer rubro, encontramos las actividades de control gerencial, donde la evaluación de costo beneficio y la rendición de cuentas son los ejes. El cuarto rubro refiere a la información y comunicación, donde la calidad y los canales de cambio, son importantes. Finalmente, el quinto rubro se refiere a la supervisión, destacándose las actividades de prevención y monitoreo, así como el seguimiento de los resultados ${ }^{18}$.

\section{De tesorería}

Según los artículos $22^{\circ}$ y $23^{\circ}$ de la Ley N. 28112 , Ley marco de la administración financiera del sector público, el Sistema Nacional de Tesorería, es el conjunto de órganos, normas, procedimientos, técnicas e instrumentos orientados a la administración de fondos públicos de las entidades y organismos del sector público, el mismo que se rige por los principios de unidad de caja y economicidad. Dicho sistema se encuentra conformado por

16 Ley N. 28411, "Ley General del Sistema Nacional de Presupuesto”, Boletín de Normas Legales del Diario Oficial El Peruano, Lima, 8 de diciembre de 2004, pp. 281887 al 281901.

17 Resolución de Contraloría N. 072-98-CG, "Normas Técnicas de Control Interno para el Sector Público," separata especial, Boletín de Normas Legales del Diario Oficial El Peruano, Lima, 18 de diciembre de 1998, pp. 11 y 12.

18 Resolución de Contraloría N. 320-2006-CG, aprueban Normas de Control Interno, Boletín de Normas Legales del Diario Oficial El Peruano, Lima, 3 de noviembre de 2006, pp. 332141 al 332157. 
la Dirección Nacional del Tesoro Público y las Oficinas de Tesorería de los pliegos presupuestarios $^{19}$.

Una de las características fundamentales de este sistema es la caja única del Tesoro Público, con el objeto de centralizar las cuentas, que deviene de los fondos de la fuente de financiamiento Recursos Ordinarios. El Tesoro Público no administra fondos distintos a la fuente de financiamiento Recursos Ordinarios, pues aquellos son administrados directamente por las entidades captadoras de fondos. El pago de obligaciones, se realiza mediante la modalidad de emisión de cheques o cartas orden o mediante abono en cuentas bancarias individuales abiertas en entidades del Sistema Financiero Nacional.

Según Ley N. ${ }^{\circ}$ 28693, Ley General del Sistema Nacional de Tesorería, se posee los principios regulatorios y las disposiciones generales para una adecuada gestión de Tesorería en la entidad pública ${ }^{20}$.

En la aplicación de los controles internos a la gestión de Tesorería, correspondía la aplicación de las siguientes normas de control interno para el sector público:

Norma 200 Normas de Control Interno para la administración Financiera Gubernamental.

Norma 230 Normas de Control Interno para el Área de Tesorería.

Norma 230-01 Unidad de Caja en la Tesorería.

230-02 Utilización del flujo de caja en la programación financiera.
230-03

Conciliaciones Bancarias.

230-04

Garantía de responsables del manejo o custodia de fondos o valores.

230-05 Medidas de seguridad para cheques, efectivos y valores.

230-06 Control y custodia de cartas fianza.

230-07 Uso de formularios membretados, pre-numerados, para el movimiento de fondos.

230-08 Uso de sello restrictivo para documentos pagados en la tesorería.

230-09 Transferencia de fondos por medios electrónicos.

230-10 Uso del fondo para pagos en efectivo $\mathrm{y} / \mathrm{o}$ fondo fijo.

230-11 Depósito oportuno en cuentas bancarias.

230-12 Arqueos de fondos y valores 230-13 Requisitos de los comprobantes de pago recibidos por entidades públicas ${ }^{21}$.

De acuerdo a la Resolución de Contraloría N. ${ }^{\circ} 320-2006-C G$, se aprueban Nuevas Normas de Control Interno, derogando las Normas antes mencionadas. En la actualidad, las entidades públicas deben elaborar sus regulaciones internas, siendo el caso para el área de Tesorería ${ }^{22}$.

Para una aplicación de los controles internos en la gestión contable, fueron aplicables las siguientes normas de control interno para el sector público:

19 Ley N.o 28112, "Ley Marco de la administración financiera del sector público, artículo $22^{\circ}$ y $23^{\circ}$ ”, Boletín de Normas Legales del Diario Oficial El Peruano, Lima, 28 de noviembre del 2003, pp. 256076.

20 Ley N.o 28693, "Ley General del Sistema Nacional de Tesorería", Boletín de Normas Legales del Diario Oficial El Peruano, Lima, 22 de marzo de 2006, pp. 315174 al 315182.

21 Resolución de Contraloría N.॰ 072-98-CG, “Aprueban Normas Técnicas de Control Interno para el Sector Público”, separata especial, Boletín de Normas Legales del Diario Oficial El Peruano, Lima, 18 de diciembre de 1998, pp. 12 y 13.

22 Resolución de Contraloría N. 320-2006-CG, "Aprueban Normas de Control Interno", Boletín de Normas Legales del Diario Oficial El Peruano, Lima, 3 de noviembre de 2006, pp. 332141 al 332157. 
Norma: $200 \quad$ Normas de control interno para la administración financiera gubernamental.

Norma: $280 \quad$ Normas de control interno para el área de contabilidad

Norma: 280-01 Aplicación de los principios y normas de contabilidad gubernamental.

280-02 Organización del sistema contable.

280-03 Conciliación de saldos.

280-04 Control de saldos pendientes de rendición y/o devolución.

280-05 Integración contable de las operaciones financieras.

280-06 Documentación sustentatoria.

280-07 Oportunidad en el registro $y$ presentación de información financiera ${ }^{23}$.

Recientemente, mediante Resolución de Contraloría N. ${ }^{\circ} 320-2006-C G$, se aprueban las Nuevas Normas de Control Interno, la misma que deroga lo anteriormente manifestado. Quedando la entidad en redactar sus propias normas internas de control ${ }^{24}$.

\section{CONCLUSIONES}

El Estado administra un flujo de efectivo, denominado "Fondos Públicos", el mismo que es el soporte cuantitativo del presupuesto institucional. Tratar de un presupuesto de ingresos y otro de gastos, es inexorablemente relacionar también de la existencia de un presupuesto cualitativo. Es decir, existen objetivos y metas que cumplir por las entidades públicas, pero éstas no se podrían cumplir sin la existencia de un presupuesto cuantitativo.

La gestión presupuestaria que deviene en la administración de un flujo de efectivo, por la naturaleza misma del ingreso y gasto, ello, obviamente será el reflejo de la gestión pública, es expuesto en los Estados Financieros. En tal sentido, se realizará la auditoría y demás acciones de control sobre la gestión institucional.

A nivel de Cuenta General de la República, se aprecia una estructura de tipo tradicional, pues la información financiera y presupuestaria está organizada según: Gobierno Central, Organismos Descentralizados Autónomos, Instituciones Públicas Descentralizadas, Fondo Consolidado de Reservas, Gobierno Regional, Bancos, Essalud, Empresas, Otras Entidades, Organismos Públicos Descentralizados del Gobierno Local, Municipalidades y Sociedades de Beneficencia Pública, tal como prescribe la Ley $\mathrm{N}^{\circ} 28708$, "Ley General del Sistema Nacional de Contabilidad”.

\section{REFERENCIAS BIBLIOGRÁFICAS}

Resolución de Contraloría Nº72-98-CG, "Aprueban Normas Técnicas de Control Interno para el Sector Público", separata especial, Boletín de Normas Legales del Diario Oficial El Peruano, Lima, 18 de diciembre de 1998, páginas 12 y 13.

Resolución de Contraloría N N 320-2006CG, "Aprueban Normas de Control Interno", Boletín de Normas Legales del Diario Oficial El Peruano, Lima, 3 de noviembre del 2006, páginas 332141 al 332157.

Resolución de Contraloría N 072-98-CG, "Aprueban normas técnicas de control interno para el Sector Público, separata especial", Boletín de Normas Legales del Diario Oficial El Peruano, Lima, 18 de diciembre de 1998, páginas 17 al 19.

Resolución de Contraloría N³20-2006CG, "Aprueban Normas de Control Interno", Boletín de Normas Legales del Diario Oficial El Peruano, Lima, 3 de noviembre del 2006, páginas 332141 al 332157.

23 Resolución de Contraloría N. 072-98-CG, "Aprueban normas técnicas de control interno para el Sector Público, separata especial”, Boletín de Normas Legales del Diario Oficial El Peruano, Lima, 18 de diciembre de 1998, pp. 17 al 19.

24 Resolución de Contraloría N. ${ }^{\circ}$ 320-2006-CG, "Aprueban Normas de Control Interno”, Boletín de Normas Legales del Diario Oficial El Peruano, Lima, 3 de noviembre de 2006, pp. 332141 al 332157. 